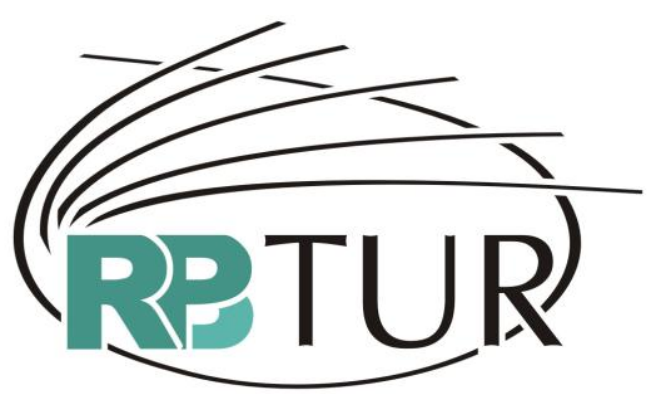

REVISTA BRASILEIRA DE PESQUISA EM TURISMO

\title{
CONHECIMENTO CIENTÍFICO PRODUZIDO NOS CURSOS DE PÓS-GRADUAÇÃO (STRICTO SENSU) EM TURISMO E ÁREAS CORRELATAS NO BRASIL NO PERIODO DE 2000 A 2006
}

\author{
SCIENTIFIC KNOWLEDGE ISSUED IN BRAZIL AT POST \\ GRADUATE PROGRAMS IN TOURISM AND CORRELATED \\ AREAS FROM 2000 TO 2006
}

\section{EL CONOCIMIENTO CIENTÍFICO PRODUCIDO EN LOS CURSOS DE POST GRADO (STRICTO SENSU) EN TURISMO Y ÁREAS AFINES EN EL BRASIL EN EL PERÍODO DE 2000 A 2006}

\author{
Christiane Fabíola Momm ${ }^{1}$ \\ Raimundo Nonato Macedo dos Santos ${ }^{2}$
}

\begin{abstract}
Resumo: investigação ${ }^{3}$ sobre o conhecimento científico produzido no campo de estudo do Turismo e áreas correlatas no Brasil, a partir das referências bibliográficas das dissertações desenvolvidas em quatro Programas de Pós-Graduação, no período de 2000 a 2006. Caracteriza-se como estudo exploratório, descritivo, qualitativo, apoiado nas ferramentas, técnicas e métodos bibliométricos e cienciométricos. 0 corpus é constituído de 334 referências bibliográficas, organizadas com base nas dissertações, e classificadas conforme o Tesauro de Turismo do Centro de

1 Bacharel em Turismo. Pós-graduada em Planejamento e Gestão do Turismo. Mestre em Ciência da Informação pelo Programa de Pós-Graduação em Ciência da Informação - PGCIN da Universidade Federal de Santa Catarina - UFSC. Email: christifabi@yahoo.com

2 Possui graduação em Engenharia Civil pela Universidade de Brasília, Mestrado em Information Stratégique Et Critique Veille Technol pela Universite d'Aix-Marseille III (Droit, Econ. et Sciences) e Doutorado em Information Stratégique et Critique Veille Technol pela Universite d'Aix-Marseille III (Droit, Econ. et Sciences). Atua como professor adjunto da Universidade Federal de Santa Catarina - UFSC. Email: raimundo@cin.ufsc.br

${ }^{3}$ Extraído de:

MOMM, C. F.. O conhecimento científico em Turismo no Brasil: cursos de pós-graduação (stricto sensu) - período de 2000 a 2006. Florianópolis, SC: UFSC, 2009. 131 f. Dissertação (Mestrado em Ciência da Informação) - Universidade Federal de Santa Catarina, Florianópolis, 2009.
\end{abstract}


MOMM, Christiane Fabíola Momm; SANTOS, Raimundo Nonato Macedo dos. Conhecimento científico produzido nos cursos de pós-graduação (stricto sensu) em turismo e áreas correlatas no Brasil no período de 2000 a 2006. Revista Brasileira de Pesquisa em Turismo. v.4, n.2, p.6485 , ago. 2010.

ISSN: $1982-6125$

Documentação Turística da Espanha. As análises geraram os seguintes resultados: constatou-se um total de 15 linhas de pesquisa no período de 2000 a 2006. Quanto ao conhecimento científico produzido, sinaliza-se que há alto grau de dispersão quanto às classes temáticas e linhas de pesquisa de todos os programas de mestrado. Demonstra a relação hierárquica entre os termos genéricos e os termos específicos e aponta as classes temáticas em que o conhecimento científico foi produzido e as áreas do conhecimento que dialogam com o campo de estudo. Finaliza com as considerações que sinalizam para reflexões acerca do desenvolvimento científico do Turismo no Brasil.

Palavras-chave: Bibliometria. Brasil. Cienciometria. Conhecimento científico. Turismo.

Abstract: a research on scientific knowledge issued in Brazil at post graduate programs in Tourism and correlated areas was conducted taking in account bibliographical references contained in Master Thesis presented in four Post Graduate Programs from 2000 to 2006. It was an exploratory, descriptive, qualitative study using bibliometric a scientometric tools and techniques. 334 bibliographical references were organized and classified following the Spanish Documental Center of Spain Tourism Thesaurus patterns. As a result 15 research lines were identified. As for scientific knowledge itself, a great diversity of research lines and issues were identified. The study demonstrates the hierarchical relation between generic terms and specific terms and points which knowledge areas have a dialogue with the field. Final considerations suggest reflection on scientific development in Tourism in Brazil is needed.

Keywords: Tourism. Bibliometrics. Scientometrics. Scientific knowledge. Brazil.

Resumen: investigación sobre el conocimiento científico producido en el campo del Turismo y áreas afines en Brasil, a partir de las referencias bibliográficas de las tesis de Maestría presentadas en cuatro Programas de Póst Graduación desde 2000 a 2006. Se trata de un estudio exporatorio, descriptivo, cualitativo, apoyado en herramientas, técnicas y métodos bibliométricos y cienciométricos. Constituyen el corpus 334 referencias bibliográficas, organizadas según las tesis y clasificadas de acuerdo al Tesauro de Turismo del Centro de Documentación Turística de España. El análisis generó el siguiente resultado: fue constatada la presencia de 15 líneas de investigación entre 2000 y 2006. En lo que respecta al conocimiento científico, se constata un alto grado de dispersión temática y de líneas de investigación en todos los programas. Se demuestra la relación jerárquica entre los términos genéricos y los específicos y se enfocan las clases temáticas en las que el conocimiento científico se produjo y aquellas que dialogan con el campo de estudio. Termina con las consideraciones que indican la necesidad de reflexión sobre el desarrollo científico del Turismo en Brasil

Palabras clave: Turismo. Bibliometría. Cienciometría. Conocimiento científico. Brasil.

\section{Introdução}

O caráter interdisciplinar do turismo permite um enfoque tanto sob o ponto de vista da atividade turística voltada para o aspecto econômico ou 
fenômeno social, quanto para o campo de estudos científicos, enquanto área de conhecimento no âmbito da educação, no meio acadêmico e científico.

No Brasil, o turismo, diferentemente do que ocorreu em outros países, passou a ser estudado a partir de 1970, com o surgimento do primeiro curso de graduação em Turismo, na cidade de São Paulo. No final da década de 1980 surgiram os primeiros cursos de pós-graduação que contemplavam esta área no Brasil.

O surgimento desses cursos, tanto em nível de graduação quanto de pós-graduação, impulsionou o desenvolvimento dos estudos turísticos e incentivou a formação de uma comunidade científica na área.

Assim, o Turismo enquanto campo de estudo no Brasil fortaleceu-se, e a produção de conhecimento científico em Turismo, em âmbito nacional, em cursos oficialmente reconhecidos pelo Ministério da Educação, exigiu o monitoramento dessa produção pela Coordenação de Aperfeiçoamento de Pessoal de Nível Superior (CAPES).

Atualmente, são recomendados seis cursos de pós-graduação stricto sensu que abarcam o Turismo e áreas correlatas, como o turismo e a hotelaria, o turismo e o meio ambiente, a hospitalidade, todos em nível de mestrado.

A existência de cursos de graduação, pós-graduação e uma entidade que monitora os cursos stricto sensu na área, remetem aos aspectos que se associam à institucionalização científica do campo de estudo. Por isso, resgatam-se alguns conceitos sobre a institucionalização de um campo de estudo tendo em vista que o turismo no Brasil já aponta para a existência de uma organização relacionada às estruturas formais, como já mencionado, por meio de seus cursos de graduação e pós-graduação lato sensu e stricto sensu.

Para Oliveira (2008, p.13), "a institucionalização de uma disciplina do conhecimento, além de suas estruturas formais sólidas, requer de seus pesquisadores e profissionais, observações, reflexões, críticas e discussões consensuais sobre o processo interno de produção do conhecimento $[\ldots]^{\prime \prime}$. 
Dessa forma, por meio do campo da Ciência da Informação e de suas metodologias específicas observou-se a possibilidade de realizar um mapeamento que identificasse em quais temáticas estão sendo desenvolvidos os estudos, e de que maneira eles poderiam evidenciar a realidade de uma determinada área e suas lacunas.

Com a investigação de como o conhecimento científico e o campo de estudo do Turismo estão se desenvolvendo nos programas de pós-graduação stricto sensu em Turismo e áreas correlatas, há a possibilidade de compreender como a produção científica está contribuindo para a institucionalização do campo e como está dialogando com outras áreas.

O objetivo geral do estudo foi investigar o campo do Turismo por meio do mapeamento da produção do conhecimento científico, considerando as dissertações dos programas de pós-graduação stricto sensu em Turismo e áreas correlatas no Brasil, no período 2000 a 2006.

Os objetivos específicos do estudo foram:

1) Recuperar as referências bibliográficas das dissertações em Turismo e áreas correlatas defendidas no Brasil, junto aos programas de pós-graduação stricto sensu recomendados pela CAPES, a partir das bases de dados e dos documentos de área, no período 2000 a 2006, e

2) Representar por meio de gráficos e mapa de visualização de conteúdo a evolução do conhecimento científico produzido sobre Turismo e áreas correlatas no Brasil, no período de 2000 a 2006.

\section{Bases Teóricas}

De acordo com Saracevic (1996, p.47), a Ciência da Informação é um "campo dedicado às questões científicas e à prática profissional voltadas para os problemas de efetiva comunicação do conhecimento e de seus registros entre os seres humanos, no contexto social, institucional ou individual do uso e das necessidades de informação". 
Dessa maneira, a Ciência da Informação pode se destacar por tratar da informação em outros campos do saber com abordagens específicas, visando atender às necessidades de informação dos usuários, a exemplo do campo de estudo do turismo.

A produção científica inerente ao corpo discente e docente destaca a importância do turismo no Brasil por meio de resultados de pesquisas científicas realizadas, envolvendo diretamente o ambiente acadêmico e, também, o ambiente organizacional.

Para Bufrem et al. (2007, p.39), ao concretizar uma reflexão sobre "um campo do conhecimento faz-se necessário pensar nas diversas possibilidades e suportes dessa produção. Considerando-se a diversidade de produtos, tais como livros, revistas científicas, teses, dissertações, monografias, comunicações em eventos, impõe-se a necessidade de categorizá-los...".

Os resultados das pesquisas científicas podem converter-se em conhecimento científico a ser disseminado entre a comunidade científica da área e entre aqueles que buscarem soluções para as atividades que desempenham. Além disso, a produção científica pode subsidiar a tomada de decisões e os pesquisadores podem encontrar, no conjunto de representações do conhecimento científico, as respostas para seus questionamentos, identificando também lacunas que requeiram maior atenção.

Para Lara (2006, p. 407), "a produção científica representa a medida do volume de livros, capítulos de livros, artigos de periódicos e outras modalidades de publicações impressas, digitais ou eletrônicas, contendo os resultados da pesquisa científica de autores, instituições, regiões, países ou áreas temáticas".

As informações provenientes das dissertações podem indicar os caminhos que estão permeando a área, o que requer uma análise adequada e, também, por conta da reduzida quantidade e mais ainda pela qualidade dos dados estatísticos coletados sobre turismo, para apoiar o trabalho dos pesquisadores. 
Nas produções científicas encontra-se um conjunto de informações que podem ser mapeadas. Para Bertin (1986, p.3) é "a partir de um conjunto de informações que se tem o ponto de partida para o tratamento da informação e para construção gráfica do que se pretende demonstrar".

A representação do conhecimento científico contribui para desenvolver a atividade e o campo de estudo em questão, de maneira sustentável. Dessa forma, observa-se o que Lage e Milone (2000, p.105) destacam: "ao profissional de turismo é imperativo um nível superior de conhecimento para utilizar a informação e torná-la conseqüente e competitiva, adequadamente inserida na aliança entre os domínios econômicos e dos enfoques mais amplos".

Ao abordar o turismo como campo de estudo é necessário compreender a estrutura referente aos programas de pós-graduação stricto sensu e as linhas de pesquisa que sinalizam a evolução no nível acadêmico, visando sua contribuição ao desenvolvimento do conhecimento científico da área.

Os cursos estão organizados de acordo com uma área de concentração e respectivas linhas de pesquisa, nas quais o corpo docente está vinculado e orienta as dissertações, assim como, os grupos de pesquisa desenvolvem seus estudos e contribuem para a construção do conhecimento científico na área.

Desde a constituição dos cursos até o período de 2006, verificou-se que os programas de mestrado apresentaram adaptações em suas linhas de pesquisa. As linhas de pesquisa foram renomeadas, conforme o quadro 1 : 
MOMM, Christiane Fabíola Momm; SANTOS, Raimundo Nonato Macedo dos. Conhecimento científico produzido nos cursos de pós-graduação (stricto sensu) em turismo e áreas correlatas no Brasil no período de 2000 a 2006. Revista Brasileira de Pesquisa em Turismo. v.4, n.2, p.6485 , ago. 2010.

ISSN: $1982-6125$

\begin{tabular}{|c|c|c|}
\hline \multicolumn{3}{|l|}{ UNIVALI/SC - Mestrado em Turismo e Hotelaria } \\
\hline Linhas de Pesquisa (LPs) & Ano & \begin{tabular}{|c|} 
LPs \\
renomeada
\end{tabular} \\
\hline Planejamento e Gestão de Destinações Turísticas & 1997 & LP1 \\
\hline Planejamento e Gestão de Empresas de Turismo & 1997 & LP2 \\
\hline Educação, Lazer e Turismo & 2000 & LP3 \\
\hline Qualificação de recursos humanos para a educação e pesquisa em turismo & 2002 & LP4 \\
\hline Planejamento e Gestão de Espaços para o Turismo & 2002 & LP5 \\
\hline \multicolumn{3}{|l|}{ UCS/RS - Mestrado em Turismo } \\
\hline Linhas de Pesquisa (LPs) & Ano & \begin{tabular}{|c|} 
LPs \\
renomeada
\end{tabular} \\
\hline Gestão Hoteleira & 2000 & LP1 \\
\hline Planejamento e Gestão do Turismo & 2000 & LP2 \\
\hline Turismo: Construções Teóricas e Modelos de Aprendizagem Social ${ }^{4}$ & 2004 & LP3 \\
\hline Turismo e Hotelaria: Organização e Gestão & 2004 & LP4 \\
\hline Turismo: Meio Ambiente, Cultura e Sociedade & 2004 & LP5 \\
\hline \multicolumn{3}{|l|}{ UAM/SP - Mestrado em Hospitalidade } \\
\hline Linhas de Pesquisa (LPs) & Ano & $\begin{array}{c}\text { LPs } \\
\text { renomeada }\end{array}$ \\
\hline Dimensões Conceituais e epistemológicas da Hospit & 2004 & LP1 \\
\hline Políticas e Gestão em Hospitalidade e Turismo & 2004 & LP2 \\
\hline \multicolumn{3}{|l|}{ UNA/MG - Mestrado em Turismo e Meio Ambiente } \\
\hline Linhas de Pesquisa (LPs) & Ano & $\begin{array}{c}\text { LPs } \\
\text { renomeada }\end{array}$ \\
\hline Planejamento e Desenvolvim & 2003 & LP1 \\
\hline Turismo e Meio Ambiente & 2003 & LP2 \\
\hline
\end{tabular}

Quadro 1: Linhas de Pesquisa Renomeadas dos Cursos de Mestrado em Turismo e Áreas Correlatas

Fonte: elaboração própria.

Para a CAPES aspectos como coerência, consistência, abrangência e atualização da área de concentração e das linhas de pesquisa são avaliados e, posteriormente, o resultado da avaliação é registrado nos documentos de área divulgados pela CAPES.

No período de desenvolvimento deste estudo observou-se que os seis cursos de pós-graduação stricto sensu em Turismo e áreas correlatas recomendados pela CAPES estavam estruturados com pelo menos duas linhas de pesquisa.

\section{Procedimentos Metodológicos e Análise dos Resultados}

4 2005: agregada às linhas Turismo e Hotelaria: Organização e Gestão e Turismo: Meio ambiente, Cultura e Sociedade. 
MOMM, Christiane Fabíola Momm; SANTOS, Raimundo Nonato Macedo dos. Conhecimento científico produzido nos cursos de pós-graduação (stricto sensu) em turismo e áreas correlatas no Brasil no período de 2000 a 2006. Revista Brasileira de Pesquisa em Turismo. v.4, n.2, p.6485, ago. 2010.

ISSN: $1982-6125$

A pesquisa caracteriza-se como exploratória, descritiva e de natureza qualitativa.

O universo da pesquisa está constituído de 334 referências bibliográficas provenientes das dissertações dos cursos de pós-graduação stricto sensu em Turismo e áreas correlatas, recomendados pela CAPES no Brasil, desenvolvidas no período de 2000 a 2006 e disponibilizadas nas bases de dados em linha.

Justifica-se a seleção deste período, pois estudos das produções científicas já foram realizados em período anterior e abordados nas teses de doutorado Pesquisa acadêmica em turismo no Brasil (1975 a 1992): configuração e sistematização documental (Rejowski, 1993) e de livre-docência Realidade turística nas pesquisas científicas: visão de pesquisadores e profissionais (Rejowski, 1997) de Rejowski, autora também da obra Turismo e pesquisa científica - Pensamento internacional x situação brasileira (1996).

A CAPES (2008) recomenda seis cursos de pós-graduação stricto sensu em nível de mestrado que estão credenciados na grande área das Ciências Sociais Aplicadas, na área de avaliação do Turismo (Administração, Ciências Contábeis e Turismo) e cujo foco de estudos desenvolve-se especificamente em Turismo. Desses cursos, um oferece mestrado profissional e cinco oferecem mestrado acadêmico, com conceitos que vão de 3 a 4, nas áreas de Turismo, Turismo e Meio Ambiente, Hospitalidade e Turismo e Hotelaria localizados nos estados de Rio Grande do Sul, Rio Grande do Norte, Distrito Federal $^{5}$, Minas Gerais, Santa Catarina e São Paulo.

Não foram considerados os cursos de mestrado credenciados e recomendados pela CAPES na grande área Multidisciplinar, por seu foco não ser específico, e por esses cursos possuírem somente uma de suas linhas de pesquisa no campo de estudo do Turismo.

Portanto, a análise e a interpretação dos resultados foram realizadas considerando-se as dissertações produzidas nos programas de mestrado em Turismo e áreas correlatas de quatro IES, listados no quadro 2 a seguir:

${ }^{5}$ Os programas de mestrado em Turismo da UFRN e da UNB iniciaram suas atividades em 2008 e no período selecionado para a realização deste estudo não apresentaram produção científica. 
MOMM, Christiane Fabíola Momm; SANTOS, Raimundo Nonato Macedo dos. Conhecimento científico produzido nos cursos de pós-graduação (stricto sensu) em turismo e áreas correlatas no Brasil no período de 2000 a 2006. Revista Brasileira de Pesquisa em Turismo. v.4, n.2, p.6485, ago. 2010.

ISSN: 1982-6125

\begin{tabular}{|l|l|l|c|}
\hline \multicolumn{1}{|c|}{ Instituição } & \multicolumn{1}{|c|}{ Local } & \multicolumn{1}{c|}{ Curso } & Início \\
\hline $\begin{array}{l}\text { Universidade do Vale do Itajaí } \\
\text { UNIVALI }\end{array}$ & $-\begin{array}{l}\text { Balneário Camboriú } \\
\text { SC }\end{array}$ & $\begin{array}{l}\text { Programa de Mestrado } 1997 \\
\text { em Turismo e Hotelaria }\end{array}$ & \\
\hline Universidade Caxias do Sul - UCS & Caxias do Sul - RS & $\begin{array}{l}\text { Programa de Mestrado } 2000 \\
\text { em Turismo }\end{array}$ & $\begin{array}{l}\text { Programa de Mestrado } 2001 \\
\text { em Hospitalidade }\end{array}$ \\
\hline $\begin{array}{l}\text { Universidade Anhembi-Morumbi } \\
\text { UAM }\end{array}$ & - São Paulo - SP & $\begin{array}{l}\text { Programa de Mestrado } 2003 \\
\text { em Turismo e Meio } \\
\text { Ambiente }\end{array}$ \\
\hline Centro Universitário UNA - UNA & Belo Horizonte - MG & \\
\hline
\end{tabular}

Quadro 2: Programas de Mestrado em Turismo e Áreas Correlatas Reconhecidos pela CAPES no Brasil na Grande Área das Ciências Sociais Aplicadas Selecionados para a Pesquisa Fonte: elaboração própria.

As técnicas e instrumentos de coleta de dados foram constituídos pelas atividades de identificação de bases de dados, acesso, recuperação, reformatação de dados e constituição de base de dados ad hoc.

A classificação da base de dados ad hoc decorreu da aplicação e representação dos termos por meio da linguagem documentária ${ }^{6}$ (LD).

$\mathrm{Na}$ classificação dos dados coletados nos repositórios em linha dos programas de mestrado e das bibliotecas em linha da CAPES e do Instituto Brasileiro de Informação em Ciência e Tecnologia (IBICT) foi utilizada a linguagem documentária, representada pelo Tesauro de Turismo (España, 2003) do Centro de Documentação Turística da Espanha (CDTE), vinculado ao Instituto de Estudos Turísticos (IET) e ao Ministério da Economia da Espanha, que pode auxiliar na recuperação da informação.

A utilização deste Tesauro de Turismo do CDTE justifica-se pela possibilidade dos termos terem abordagem mais específica, enquanto que o Tesauro de Turismo e Lazer da OMT possui termos em abrangência global e está estruturado de forma geral. Além disso, a utilização do Tesauro de Turismo do CDTE fez-se pela ausência de um documento no Brasil, que fosse dotado das mesmas características do Tesauro selecionado. A sua organização compreende uma hierarquia representada por classes temáticas que correspondem aos termos maiores, os termos genéricos (TGs) e, por fim, os termos específicos (TEs).

${ }^{6}$ Linguagem documentária é a linguagem utilizada para representar o conteúdo de uma determinada área de pesquisa. Neste estudo a linguagem documentária utilizada foi o Tesauro (do inglês, Thesaures). 
De acordo com o Tesauro de Turismo do CDTE (2003, p. 117-123, tradução nossa), os termos maiores que compreendem as classes temáticas constituem-se das categorias e respectivas subcategorias listadas abaixo:

a) Obras de referência: bibliografias, anuários, biblioteconomia, manuais, dicionários, enciclopédias e teses;

b) Organizações turísticas: organismos públicos, organismos privados, organismos internacionais, estatutos;

c) Turismo e meio social: história do turismo, filosofia do turismo, psicologia do turismo, sociologia do turismo, antropologia do turismo, saúde e higiene, meios de comunicação, literatura turística e investigação turística;

d) Patrimônio turístico: geografia turística, patrimônio cultural, patrimônio natural e arquitetura e urbanismo;

e) Atividades esportivas e recreativas: esportes, campismo, excursionismo, turismo equestre, cicloturismo, parques recreativos, naturismo, turismo de montanha;

f) Serviços turísticos: meios de hospedagem, hotelaria, classificação de estabelecimentos turísticos, restauração, gastronomia, agência de viagens, transportes, novas tecnologias, salas de diversão e espetáculos;

g) Economia do turismo: estudos econômicos, contas nacionais de turismo, empresas, financiamento, tributos, trabalho, comércio e consumo, competitividade turística, seguros;

h) Política turística: fomento do turismo, planejamento turístico, controle turístico; modalidades turísticas, cooperação internacional, coordenação estatal, marketing e promoção;

i) Direito: direito do turismo, legislação administrativa turística, convênios laborais, jurisprudência do turismo;

j) Educação e formação turística: formação turística, organismos docentes, cursos de pós-graduação, bolsas, currículo educacional, titulações.

A utilização de um Tesauro de Turismo possibilitou a representação do conteúdo informacional das dissertações produzidas nos programas de mestrado pautando-se nos títulos, resumos e palavras-chave propostas pelos autores. Os termos maiores, representados pelas classes temáticas, termos genéricos e termos específicos fundamentaram a classificação da base de dados e auxiliaram na formulação da análise, interpretação e validação dos resultados obtidos.

Para representar por meio de gráficos e mapa de visualização do conteúdo a evolução do conhecimento científico em Turismo no Brasil, foram utilizados procedimentos cienciométricos, o que permitiu a análise descritiva. 
De acordo com Macias-Chapula (1998), a cienciometria é um método quantitativo utilizado para analisar o comportamento e as atividades da ciência, partindo-se da sua produção e comunicação. O pesquisador utiliza-se de técnicas avançadas que possibilitam a análise de dados bibliográficos das teses e dissertações, permitindo o mapeamento dos temas de pesquisa estudados na área e, posteriormente, o desenvolvimento do conhecimento científico (ELIEL, 2007).

Assim, assume-se que a análise dos dados foi cienciométrica, descritiva e interpretativa.

Quanto à classificação, com base no Tesauro de Turismo do CDTE, alguns termos do idioma espanhol sofreram adaptações na tradução para o idioma português (Brasil), pois, dessa forma, houve a possibilidade de classificar os termos genéricos (TGs) e os termos específicos (TEs).

Os softwares utilizados foram inicialmente o Excel, para a formulação da base de dados e dos gráficos e, para a elaboração do mapa de visualização, o DataView e o Statistica 6.0.

Quanto às limitações da pesquisa, alguns aspectos podem ser considerados, dentre eles:

a) Os dados disponíveis nas bases institucionais não foram previstos para a realização de estudos cienciométricos, requerendo para isso exaustivo trabalho manual de coleta e tratamento das estruturas de dados (reformatação);

b) A limitação de dados disponíveis nas bases de dados em linha das IES exigiu e demandou um tempo superior ao estimado;

c) Os dados coletados referentes aos resumos e descritores elaborados pelos autores das dissertações apresentaram inconsistências, o que também ocasionou um tempo superior ao estimado, na organização e classificação das referências bibliográficas;

d) As informações recuperadas nos repositórios em linha e/ou nos documentos de área da CAPES não apresentavam aderência quanto ao conteúdo 
ISSN: $1982-6125$

disponibilizado, podendo suscitar questionamentos quanto à validade de seus conteúdos.

Quanto à análise e interpretação dos resultados e contribuições do estudo realizado, observou-se que a área de pesquisa do turismo no Brasil apresentou-se no período de 2000 a 2006, da seguinte maneira:

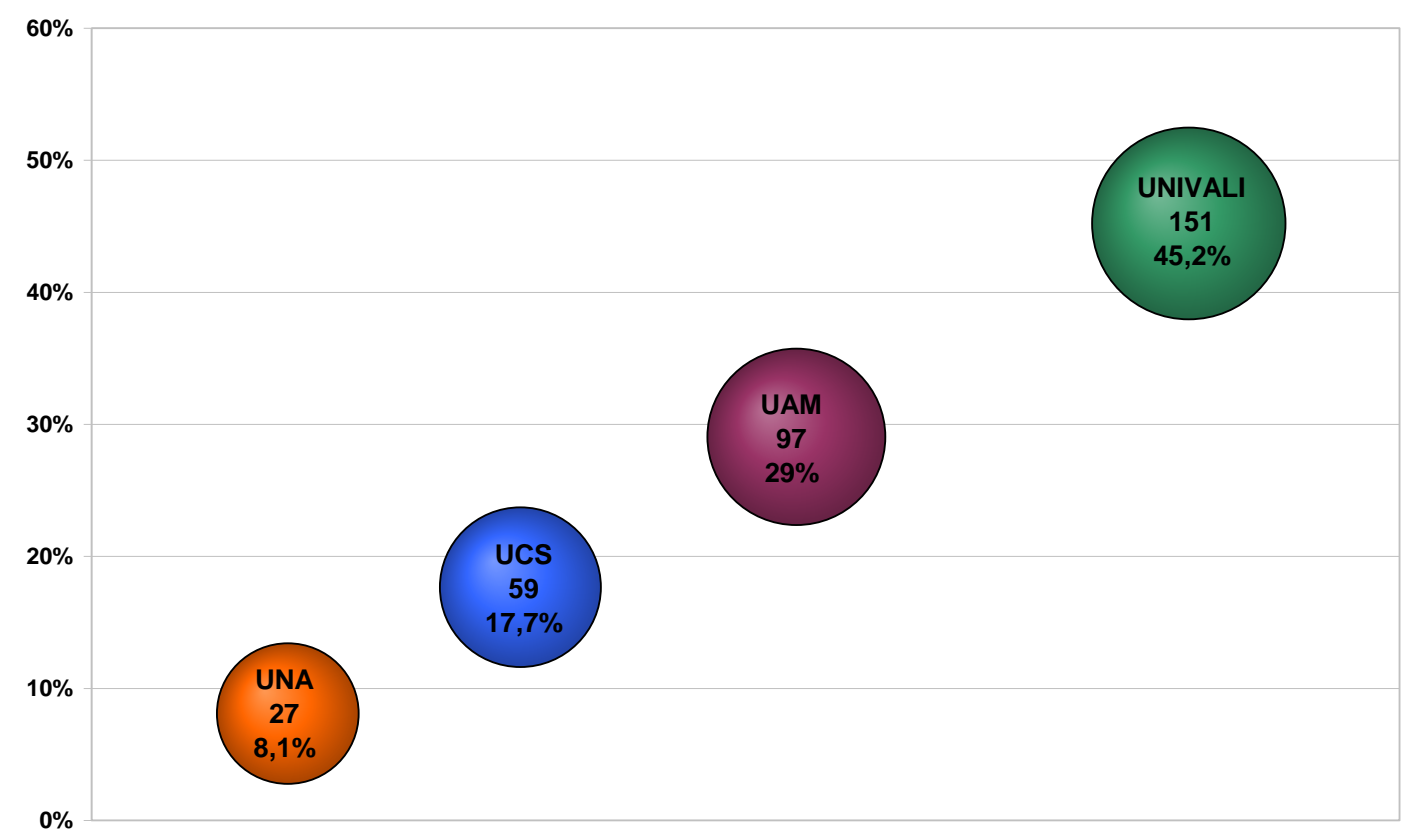

Figura 1: Total de Dissertações dos Cursos de Mestrados Stricto Sensu em Turismo e Áreas Correlatas no Brasil: Período de 2000 a 2006 Fonte: elaboração própria.

A figura 1 aponta a quantidade de dissertações produzidas no período de 2000 a 2006 nos cursos de mestrado em Turismo e Hotelaria da UNIVALI/SC, Turismo da UCS/RS, Hospitalidade da UAM/SP e Turismo e Meio Ambiente da UNA/MG.

O programa de mestrado em Turismo e Hotelaria da UNIVALI/SC, reconhecido em 1997 pela CAPES, possui 45,2\% das dissertações produzidas.

Na sequência está a produção do curso de mestrado em Hospitalidade da UAM/SP, com 29\% das dissertações produzidas. Iniciou suas atividades efetivas em 2002, um ano após o programa de mestrado da UCS/RS, e ofereceu uma quantidade maior de vagas. 
ISSN: $1982-6125$

A UCS/RS, que foi reconhecida pela CAPES, em 2000, e iniciou suas atividades efetivamente em 2001, apresentou 17,7\% das dissertações.

Por último, foi pesquisado o curso de mestrado em Turismo e Meio Ambiente da UNA/MG, reconhecido em 2003, com 8,1\% das dissertações.

A produção do conhecimento científico em Turismo e áreas correlatas no Brasil, pode ser visualizado a partir da relação existente entre as classes temáticas dispostas no Tesauro de Turismo e as linhas de pesquisa (ver quadro 1) dos cursos de mestrado selecionados. Para observar essa relação, utilizouse o coeficiente de correlação de Jaccard, que considera a relação entre a presença e a ausência dos termos a saber: 
MOMM, Christiane Fabíola Momm; SANTOS, Raimundo Nonato Macedo dos. Conhecimento científico produzido nos cursos de pós-graduação (stricto sensu) em turismo e áreas correlatas no Brasil no período de 2000 a 2006. Revista Brasileira de Pesquisa em Turismo. v.4, n.2, p.6485 , ago. 2010.

ISSN: $1982-6125$

0,4

UNIVALI - LP3 / PATRIMONIO TURISTICO UCS - LP3 / PATRIMONIO TURISTICO UNA - LP2 / POLITICA TURISTICA UAM - LP1 / ECONOMIA DO TURISMO UNIVALI - LP4 / POLITICA TURISTICA UCS - LP3 / POLITICA TURISTICA UNA - LP1 / SERVICO TURISTICO LP15 / POLITICA TURISTICA UNA - LP2 / SERVICO TURISTICO UNIVALI - LP1 / SERVICO TURISTICO UAM - LP2 / DIREITO UCS - LP2 / SERVICO TURISTICO UNIVALI - LP2 / ORGANIZACAO TURISTICA UNA - LP1 / ECONOMIA DO TURISMO UCS - LP5 / ECONOMIA DO TURISMO UNIVALI - LP5 / TURISMO E MEIO SOCIAL UCS - LP3 / ECONOMIA DO TURISMO UNIVALI - LP2 / DIREITO UAM - LP1 / POLITICA TURISTICA UCS - LP2 / PATRIMONIO TURISTICO UCS - LP1 / ECONOMIA DO TURISMO UCS - LP1 / PATRIMONIO TURISTICO UNIVALI - LP5 / ORGANIZACAO TURISTICA UCS - LP2 / TURISMO E MEIO SOCIAL UNIVALI - LP5 / ECONOMIA DO TURISMO UNIVALI - LP5 / ATIV. ESPORTIVA E UNIVALI - LP5 / ATIV. ESPORTIVA E
UCS - LP5 / POLITICA TURISTICA UAM - LP2 / EDUC. E FORMACAO TURISTICA UCS - LP3 / TURISMO E MEIO SOCIAL UNIVALI - LP4 / TURISMO E MEIO SOCIAL UCS - LP2 / POLITICA TURISTICA UNA - LP1 / EDUC. E FORMACAO TURISTICA UAM - LP1 / ATIV. ESPORTIVA E RECREATIVA UCS - LP5 / SERVICO TURISTICO

UNIVAL - LP1 / PATRIMONIO TURISTICO UCS - LP4 / POLITICA TURISTICA UCS - LP4 / TURISMO E MEIO SOCIAL UCS - LP4 / DIREITO UNIVALI - LP2 / POLITICA TURISTICA UNA - LP2 / ECONOMIA DO TURISMO UNIVALI - LP1 / POLITICA TURISTICA UCS - LP2 / ECONOMIA DO TURISMO UNIVALI - LP5 / SERVICO TURISTICO UCS - LP5 / ORGANIZACAO TURISTICA UCS - LP5 / ORGANIZACAO TURISTICA UNA - LP1 / TURISMO E MEIO SOCIAL UCS - LP5 / TURISMO E MEIO SOCIAL UCS - LP4 / EDUC. E FORMACAO TURISTICA
UNIVALI - LP2 / PATRIMONIO TURISTICO UNIVALI - LP2 / EDUC. E FORMACAO TURISTICA UAM - LP2 / PATRIMONIO TURISTICO UAM - LP1 / ORGANIZACAO TURISTICA UAM - LP2 / ORGANIZACAO TURISTICA UCS - LP5 / EDUC. E FORMACAO TURISTICA UNA - LP1 / PATRIMONIO TURISTICO UCS - LP5 / PATRIMONIO TURISTICO UNA - LP1 / POLITICA TURISTICA UNIVALI - LP1 / EDUC. E FORMACAO TURISTICA UNIVALI - LP1 / ATIV. ESPORTIVA E UAM - LP2 / TURISMO E MEIO SOCIAL UNIVALI - LP1 / TURISMO E MEIO SOCIAL UCS - LP3 / ORGANIZACAO TURISTICA UAM - LP1 / PATRIMONIO TURISTICO UCS - LP4 / PATRIMONIO TURISTICO UCS - LP4 / PATRIMONIO TURISTICO
UCS - LP4 / ECONOMIA DO TURISMO UCS - LP4 / ECONOMIA DO TURISMO
UAM - LP2 / POLITICA TURISTICA UAM - LP2 / POLITICA TURISTICA
U - LP2 / PATRIMONIO TURISTICO UNA - LP2 / PATRIMONIO TURISTICO UCS - LP4 / SERVICO TURISTICO UNIVALI - LP4 / EDUC. E FORMACAO TURISTICA UNIVALI - LP3 / EDUC. E FORMACAO TURISTICA UNIVALI - LP2 / ECONOMIA DO TURISMO

UAM - LP1 / EDUC. E FORMACAO TURISTICA UNIVALI - LP2 / TURISMO E MEIO SOCIAL UNIVALI - LP2 / TURISMO E MEIO SOCIAL
UAM - LP1 / TURISMO E MEIO SOCIAL UAM - LP2 / SERVICO TURISTICO UNIVALI - LP2 / SERVICO TURISTICO UAM - LP2 / ECONOMIA DO TURISMO UNIVALI - LP5 / PATRIMONIO TURISTICO UNIVALI - LP5 / POLITICA TURISTICA
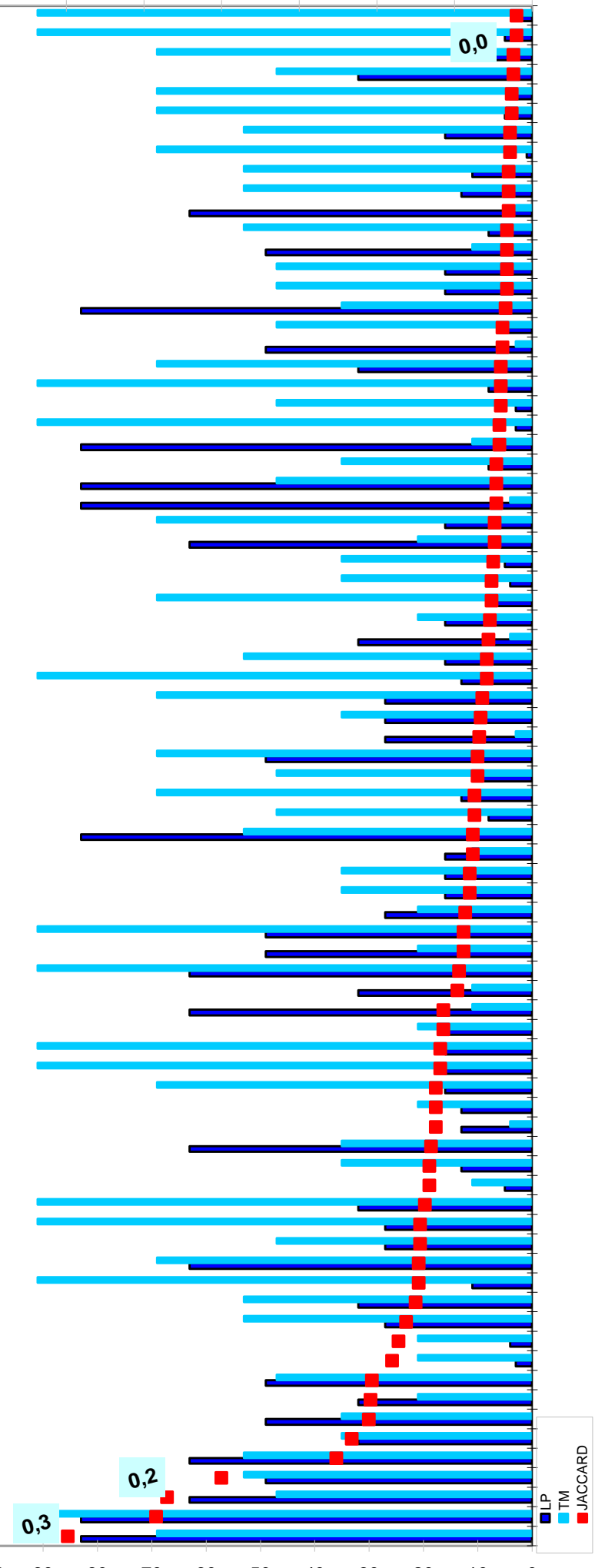

$$
\begin{array}{lllllllllll}
100 & 90 & 80 & 70 & 60 & 50 & 40 & 30 & 20 & 10 & 0
\end{array}
$$

Figura 2: Correlação entre as Linhas de Pesquisa e as Classes Temáticas nos Cursos de Mestrado

Fonte: elaboração própria. 
Ao representar todas as linhas de pesquisa do período de 2000 a 2006, dos quatro programas de mestrado selecionados, observa-se dispersão e ausência de classes temáticas consolidadas, desenvolvidas nas dissertações.

A figura 2 demonstra que, de um total de dez classes temáticas apresentadas no Tesauro de Turismo do CDTE, os maiores índices de correlação pertencem às classes temáticas Política Turística e Patrimônio Turístico associadas à linha de pesquisa LP5 da UNIVALI/SC, com índice de correlação de 0,3, e as classes Economia do Turismo associada a LP2 da UAM/SP, Serviços Turísticos associado às LP2 da UAM/SP e LP2 da UNIVALI/SC com índice de correlação de 0,2.

Os baixos índices de correlação reforçam a dispersão na área de pesquisa e sinalizam a necessidade de reflexão sobre as descrições das linhas de pesquisa dos cursos de Mestrado. As avaliações da CAPES retratam a análise sobre a estrutura do curso, área de concentração e linhas de pesquisa, produção intelectual docente e discente, atividades de pesquisa, currículo do curso e se estendem até a formação dos docentes que atuam nos cursos.

Por isso, resgata-se o papel das instituições e associações de representatividade. A Associação Nacional de Pesquisa e Pós-Graduação em Turismo (ANPTUR) realiza, desde o ano 2002, um evento científico que reúne os docentes e discentes dos programas de Mestrado em Turismo e em áreas correlatas para avaliar o desenvolvimento científico e promover o intercâmbio na área. Os docentes, os pesquisadores e os discentes que atuam nos cursos de Mestrado e os profissionais que desenvolvem pesquisas podem direcionar os rumos do desenvolvimento do conhecimento científico em Turismo no Brasil.

Assim, para observar se os baixos índices de correlação são recorrentes, optou-se por aprofundar a representação dos conteúdos das dissertações demonstrando os termos genéricos de nível 1 (subcategorias das classes temáticas) relacionando-as às linhas de pesquisa, conforme a figura 3 : 
MOMM, Christiane Fabíola Momm; SANTOS, Raimundo Nonato Macedo dos. Conhecimento científico produzido nos cursos de pós-graduação (stricto sensu) em turismo e áreas correlatas no Brasil no período de 2000 a 2006. Revista Brasileira de Pesquisa em Turismo. v.4, n.2, p.6485, ago. 2010.

ISSN: $1982-6125$

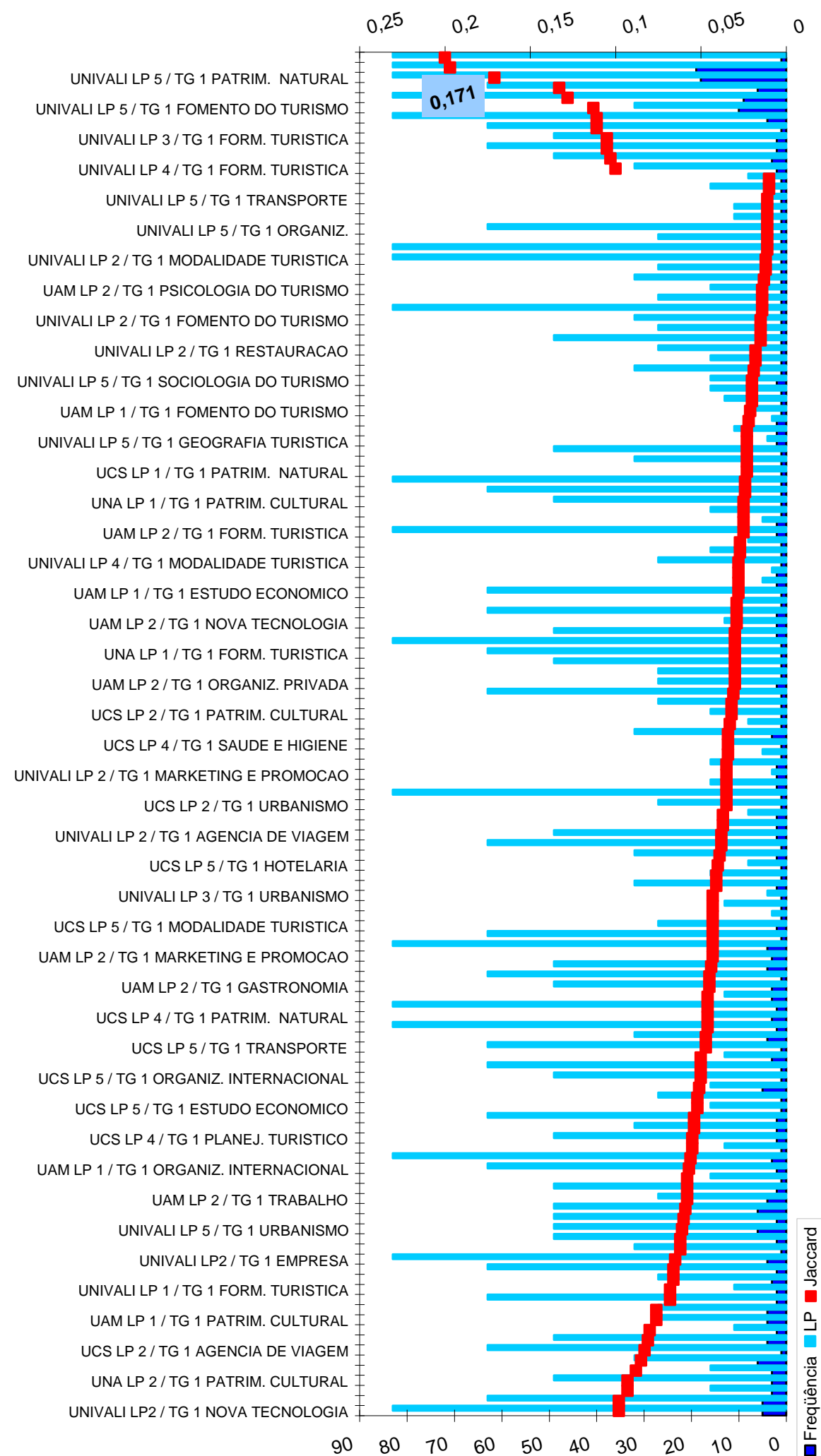

Figura 3: Correlação entre as Linhas de Pesquisa e os Termos Genéricos de Nível 1 Fonte: elaboração própria. 
A figura 3 aponta os termos genéricos de nível 1 (subcategorias das classes temáticas) que foram 0 foco dos estudos desenvolvidos nas dissertações produzidas e que se associam com as linhas de pesquisa dos programas de mestrado selecionados.

O índice de correlação próximo de 0,2 confirma que se destacam os termos genéricos de nível 1 patrimônio natural e fomento do turismo associados à LP5 da UNIVALI/SC. Esses termos genéricos de nível 1 compõem as classes temáticas Patrimônio Turístico e Política Turística que, por sua vez, são as classes temáticas com maior grau de inserção na LP5 da UNIVALI/SC.

Mesmo apresentando um índice de correlação considerado alto em relação aos demais apresentados na figura, pode-se inferir que a dispersão é predominante. A aplicação desses métodos e técnicas bibliométricas, bem como, a aplicação de um Tesauro específico da área de pesquisa do Turismo, reforçam o estudo voltado para o estado da arte da área, sinalizando as lacunas representadas pela dispersão e desequilíbrio no desenvolvimento do conhecimento científico na área. Todos os programas de Mestrado selecionados apontaram um alto grau de dispersão, não só no que se refere aos termos genéricos e termos específicos, assim como, nas classes temáticas.

Entretanto, considerando-se o aprofundamento para representar por meio de termos específicos o conteúdo das dissertações produzidas, optou-se por analisar a relação hierárquica entre os TGs de nível 1 e os TEs de níveis 1 , 2,3 e 4 , conforme se demonstra na figura 4 : 
MOMM, Christiane Fabíola Momm; SANTOS, Raimundo Nonato Macedo dos. Conhecimento científico produzido nos cursos de pós-graduação (stricto sensu) em turismo e áreas correlatas no Brasil no período de 2000 a 2006. Revista Brasileira de Pesquisa em Turismo. v.4, n.2, p.6485, ago. 2010.

ISSN: $1982-6125$

Tree Diagram for Variables

Ward's method

1-Pearson $r$

TG 1 MEIO DE HOSPEDAGEM

TE 1 MEIO DE HOSPEDAGEMIPRIVADO]

TG 1 COMERCIO E CONSUMO

TE 1 TURISMO DE NEGOCIOS

TG 1 CONTA NACIONAL DE TURISMO

TE 1 SISTEMA NACIONAL DE CONTABILIDADE

TG 1 ESTUDO ECONOMICO

TE 1 IMPACTO ECONOMICO

TE 1 DESENVOLVIMENTO TURISTICO

TG 1 GASTRONOMIA

TE 1 ENOLOGIA TG 1 TRABALHO
TE 1 PROFISSAO DO TURISMO
TG 1 HOTELARIA

TE 1 GESTAO HOTELEIRA

TG 1 MARKETING E PROMOCAO

TG 1 MEIO DE COMUNICACAO

TE 1 IMPRENSA

TG 1 PESQUISA TURISTICA

TE 1 ESTATISTICA

TG 1 SISTEMA DE TRANSPORTE

TE 1 TRANSPORTE

TG 1 NOVA TECNOLOGIA

TE 1 TURISMO E INFORMATICA

T 1 PLANEJAMENTO TURISTICO

TE 1 ORDENACAO TURISTICA TERRITORIAL

TG 1 DEMOGRAFIA
TE 1 ANT ROPOLOGIA DO TURISMO

TG 1 ESPORTE

TE 1 ESPORTE NAUTICO

TG 1 CONTROLE TURISTICO

TE 1 CONTROLE POLICIAL

TG 1 MEIO AMBIENTE

TE 1 PATRIMONIO NATURAL

TG 1 MERCADO TURISTICO

TE 1 ESTUDO ECONOMICO

TG 1 PROMOCAO TURISTICA

TE 1 MARKETING E PROMOCAO

TE 1 TURISMO NAUTICO

TE 1 TURISMO RURAL

TG 1 PATRIMONIO CULTURAL

TE 1 ETNOLOGIA

TG 1 URBANISMO

TE 1 ARQUITETURA E URBANISMO

TE 2 INFRA-ESTRUTURA URBANA

TG 1 EMPRESA

TE 1 GESTAO E ADMINISTRACAO DE EMPRESA

TE 1 EMPRESA PRIVADA

TE 1 CONTROLE DE QUALIDADE
TG 1 RESTAURACAO

TE 1 ESTRUTURA DE RESTAURACA

TE 1 SERVICO DE COMIDA

TE 1 GESTAO DA RESTAURACAO

TG 1 SOCIOLOGIA DO TURISMO

TE 1 TURISMO SOCIAL

TE 1 CUSTO SOCIAL

TG 1 TRANSPORTE

TE 1 SISTEMA DE TRANSPORTE-

TE 2 TRANSPORTE TERRESTRE

TE 3 TRANSPORTE FERROVIARIO

TE 2 TRANSPORTE AEREO

ATRIMONIO NATURAL

TE 1 MEIO AMBIENTE

TE 2 TUR

TE 3 TURISMOSUSTENTAVE

TE 2 IMPACTO AMBIENTAL

TE 1 GEOMORFOLOGIA

TE 1 RECURSONATURAL

TE 2 GESTAO AMBIENTAL
TE 3 CONSERVACAO DO MEIO AMBIENTE

TE 4 AREA PROTEGIDA
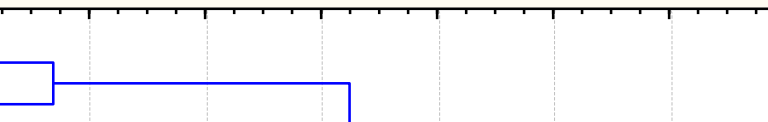
MOMM, Christiane Fabíola Momm; SANTOS, Raimundo Nonato Macedo dos. Conhecimento científico produzido nos cursos de pós-graduação (stricto sensu) em turismo e áreas correlatas no Brasil no período de 2000 a 2006. Revista Brasileira de Pesquisa em Turismo. v.4, n.2, p.6485, ago. 2010.

ISSN: $1982-6125$

Foram analisadas as relações entre as classes temáticas e as linhas de pesquisa dos programas de mestrado selecionados, bem como, os termos genéricos de nível 1 e as linhas de pesquisa. Por fim, optou-se por representar em um mapa de visualização na forma de dendograma a relação hierárquica existente entre os termos genéricos de nível 1 e seus termos específicos nos níveis $1,2,3$ e 4.

As classes temáticas coordenam a organização do campo de estudo, no entanto, é necessário atentar para as relações que se estabelecem entre as classes temáticas e os TGs, até chegar aos TEs (especificação do assunto abordado no campo de estudo do Turismo).

A representação se faz a partir da relação de proximidade e distanciamento dos termos em associação com as frequências em que ocorreram. Exemplo: TG 1 Patrimônio Natural, pertencente à classe temática Patrimônio Turístico, é recorrente quando associado com os termos específicos de nível 1 e de nível 2 Meio Ambiente, e Turismo e Meio Ambiente, respectivamente.

Outros TGs em nível 1 e os TEs 1 , 2 e 3, que não se relacionaram com maior frequência nas classificações/representações de conteúdo, formam blocos médios. Nos grandes blocos, localizados na parte média inferior do mapa de visualização, evidenciam-se as relações de termos genéricos de nível 1 e termos específicos de nível 1, 2 e 3 pertencentes às classes temáticas (termos maiores) Patrimônio Turístico, Política Turística, Serviços Turísticos e Economia do Turismo.

E ainda explicita-se pelas relações demonstradas no dendograma, as áreas que dialogam com o campo de estudo do turismo, quais sejam, a administração, a economia, o direito, a antropologia, a sociologia, a ecologia, transportes, dentre outras.

Todos os resultados apresentados no gráfico e mapa de visualização na forma de dendograma sinalizaram a concentração dos estudos desenvolvidos nas dissertações em somente quatro classes temáticas (de um total de dez 
MOMM, Christiane Fabíola Momm; SANTOS, Raimundo Nonato Macedo dos. Conhecimento científico produzido nos cursos de pós-graduação (stricto sensu) em turismo e áreas correlatas no Brasil no período de 2000 a 2006. Revista Brasileira de Pesquisa em Turismo. v.4, n.2, p.6485 , ago. 2010.

ISSN: $1982-6125$

classes temáticas elencadas no Tesauro de Turismo do CDTE), quais sejam Patrimônio Turístico, Política Turística, Economia do Turismo e Serviços Turísticos.

\section{Considerações}

A investigação sobre o conhecimento científico produzido nos programas de pós-graduação stricto sensu em Turismo e áreas correlatas, no período de 2000 a 2006, apresentou, por meio da análise dos gráficos e do mapa de visualização, pautadas nas bases teóricas e nos documentos de área da CAPES, os seguintes resultados:

- o programa de mestrado em Turismo e Hotelaria da UNIVALI/SC possui a maior parcela das dissertações produzidas no campo de estudo, seguido do mestrado em Hospitalidade da UAM/SP e do mestrado em Turismo da UCS/RS, seguido do mestrado em Turismo e Meio Ambiente da UNA/MG;

- para observar a consistência e coerência do Tesauro de Turismo do CDTE em relação à institucionalização cognitiva do campo representada pelas linhas de pesquisa em associação com as classes temáticas que se destacaram, verificou-se que há predominância hierárquica das classes temáticas Patrimônio Turístico, Política Turística, Economia do Turismo e Serviços Turísticos em relação às classes Organização Turística, Atividade Esportiva e Recreativa, Direito, Turismo e Meio Social e Educação e Formação Turística;

- as linhas de pesquisa relacionadas com os TGs 1 validaram o alto índice de dispersão apresentado entre as linhas de pesquisa e as classes temáticas;

- a hierarquia é predominante não só no que se refere às classes temáticas, assim como, no que se refere aos termos genéricos TGs de nível 1 e em todos os níveis dos termos específicos (TEs). O campo de estudo do Turismo no Brasil dialoga com as áreas da Sociologia, Antropologia, 
MOMM, Christiane Fabíola Momm; SANTOS, Raimundo Nonato Macedo dos. Conhecimento científico produzido nos cursos de pós-graduação (stricto sensu) em turismo e áreas correlatas no Brasil no período de 2000 a 2006. Revista Brasileira de Pesquisa em Turismo. v.4, n.2, p.6485 , ago. 2010.

ISSN: $1982-6125$

Geografia, Arquitetura e Urbanismo, Administração, Economia, Direito dentre outros.

Considera-se que, para se consolidar a institucionalização científica do campo de estudo do Turismo no Brasil, é necessário que haja reflexões e reestruturações no campo de estudo por parte do meio acadêmico e demais profissionais responsáveis por desenvolver pesquisas no campo de estudo do Turismo.

\section{Referências}

BERTIN, J.. As etapas da decisão. In: BERTIN, J.. A neográfica e o tratamento gráfico da informação. Curitiba: Editora UFPR, 1986. p.2-11

BRASIL. Coordenação de Aperfeiçoamento de Pessoal de Nível Superior CAPES. Cursos recomendados e reconhecidos. Disponível em: http://www.capes.gov.br . Acesso em: 29 abr.2008.

BUFREM, L. et al. Produção científica em ciência da informação: análise temática em artigos de revistas brasileiras Perspectivas em Ciência da Informação. Belo Horizonte, v.12, n.1, p. 38-49, jan./abr. 2007. Disponível em: $\quad<$ http://www.scielo.br/scielo.php?script=sci_arttext\&pid=S141399362007000100004\&lng=\&nrm=iso\&tlng=> Acesso em: 24 fev. 2008.

ELIEL, R. A.. Institucionalização da ciência da informação no Brasil: estudo da convergência entre a produção científica e os marcos regulatórios da área. Campinas, SP: PUC - Campinas, 2007. 84 f. Dissertação (Mestrado em Ciência da Informação) - Pontifícia Universidade Católica de Campinas, Campinas, 2007.

ESPAÑA. Ministerio de Economía y Centro de Documentación Turistica de España - CDTE. Tesauro - Turismo. Madrid, España: CDTE, 2003.

LAGE, B. H. G.; MILONE, P. C.. Turismo: Teoria e Prática. São Paulo: Atlas, 2000.

LARA, M. L. G. de. Termos e conceitos da área de comunicação e produção Científica. In: POBLACIÓN, D. A.; WITTER, G. P.; SILVA, J. F. M. da. Comunicação \& produção científica: contexto, indicadores e avaliação. São Paulo: Angellara, 2006. Glossário. p.387-414.

MACIAS-CHAPULA, C. A.. O papel da informetria e da cienciometria e sua perspectiva nacional e internacional. Ciência da Informação, Brasília, v. 27, n. 2, p. 134-140, maio/ago. 1998. Disponível em: http://www.scielo.br/scielo.php?pid=S010019651998000200005\&script=sci abstract\&tlng=pt . Acesso em: 27 set. 2007. MOMM, C. F.. O conhecimento científico em Turismo no Brasil: cursos de pósgraduação (stricto sensu) - período de 2000 a 2006. Florianópolis, SC: UFSC, 
2009. 131 f. Dissertação (Mestrado em Ciência da Informação) - Universidade Federal de Santa Catarina, Florianópolis, 2009..

OLIVEIRA, M. J. de. Institucionalização da Pesquisa Científica: estudo do repertório metodológico das dissertações defendidas em dois Cursos de PósGraduação em Ciência da Informação no Brasil. Campinas, SP: PUC-Campinas, 2008. 122 f. Dissertação (Mestrado em Ciência da Informação) - Pontifícia Universidade Católica de Campinas, Campinas, 2008.

REJOWSKI, M.. Realidade turística nas pesquisas científicas: visão de pesquisadores e profissionais. São Paulo, SP: ECA - USP, 1997. Tese de Livre Docência. (Livre Docência) - Escola de Comunicações e Artes/ECA Universidade de São Paulo/USP. São Paulo, 1997. 2 v.

REJOWSKI, M.. Turismo e pesquisa científica: pensamento internacional $\mathrm{x}$ situação brasileira. Campinas: Papirus, 1996.

REJOWSKI, M.. Pesquisa acadêmica em turismo no Brasil (1975 a 1992): configuração e sistematização documental. São Paulo, SP: 1993. Tese. (Doutorado em Ciências da Comunicação) - Escola de Comunicações e Artes/ECA, Universidade de São Paulo/USP. São Paulo, 1993. 2 v.

SARACEVIC, T.. Ciência da informação: origem, evolução e relações. Perspectivas em Ciência da Informação, Belo Horizonte, v. 1, n. 1, p .41-62, jan./jun. 1996.

Artigo recebido em abril de 2010.

Aprovado para publicação em agosto de 2010. 